\title{
"Choosing Wisely Canada" and antimicrobial stewardship: A shared focus on reducing unnecessary care
}

\author{
Born $\mathrm{KB}^{1^{*}}$, Leis $\mathrm{JA}^{2}$, Gold $\mathrm{WL}^{3}$, Levinson $\mathrm{W}^{1}$ \\ ${ }^{1}$ Choosing Wisely Canada, University of Toronto, Toronto, ON \\ 2 Division of Infectious Diseases and Centre for Quality Improvement and Patient Safety, University of Toronto, Toronto, ON \\ ${ }^{3}$ Division of Infectious Diseases, University of Toronto, Toronto, ON
}

*Correspondence: karen.born@mail.utoronto.ca

\begin{abstract}
There is a growing movement in medicine which recognizes that some tests, treatments or procedures do not add value for patients, and may even cause harm. The "Choosing Wisely Canada" campaign is a grassroots, physician-led campaign to engage physicians and patients in conversations about overuse of unnecessary tests, treatments and procedures to improve the quality of health care. This article reviews the underlying principles of this campaign and its spread across Canada. It also highlights the alignment between the principles of Choosing Wisely Canada with those of antimicrobial stewardship, which share similar motivations, challenges and opportunities.
\end{abstract}

\section{Introduction}

Improving quality, safety and patient centeredness are common objectives of health care providers, organizations and systems across Canada. Canadian and international studies have demonstrated the ubiquity of adverse events and harm associated with medical care $(1,2)$. The patient safety movement has highlighted that some of this harm is preventable and has implemented a variety of strategies such as checklists, bundles, improved measurement and continuing professional development programs. An important target in the patient safety movement is preventing unnecessary care, defined as medical care being delivered to patients that provides no benefit, or lacks a clinical indication. When care provides no benefit or is not clinically indicated, the quality improvement target becomes unnecessary care itself. Unnecessary care is driven by a number of complex forces such as established practice habits, time pressures, discomfort with diagnostic uncertainty and perceived patient expectations.

Although it may be well-intentioned, unnecessary care can trigger a "cascade effect" of further tests, procedures and treatments that can cause physical and psychological harms (3). Further investigation can lead to adverse events, complications, as well as heightened patient stress and anxiety. Unnecessary care also strains already stretched health care budgets. Data from the United States reports that $30 \%$ of health care spending is wasteful (4). While there is no similar global figure in Canada, there is rapidly expanding literature demonstrating the pervasiveness of unnecessary tests, treatments and procedures (5).

While physicians and patients agree that improving quality of care and curbing overuse of unnecessary tests, treatments and procedures is an important goal, changing physician behaviours and patient expectations is challenging and complex. The "Choosing Wisely Canada" campaign is aimed at addressing these complex causes by providing evidence-based educational materials to both physicians and patients, by encouraging a conversation about making informed choices, and by facilitating a broader public dialogue around unnecessary care. The goal of this article is to review the underlying principles of this campaign and to highlight the alignment between the principles of Choosing Wisely Canada with those of antimicrobial stewardship. 


\section{Choosing Wisely Canada: A physician-led campaign}

Choosing Wisely Canada was launched in April 2014. It was modelled upon the American Board of Internal Medicine Foundation Choosing Wisely campaign launched in April 2012 that encourages physicians and patients to talk about unnecessary tests, treatments and procedures to improve the value of care, and reduce harm.

To date, 45 Canadian specialty societies have joined the campaign. Participating societies commit to developing a "Top 5 list of tests and treatments physicians and patients should question." These recommendations state what physicians should stop doing; there are over 100 recommendations at present and more are about to be released.

\section{"More medicine is not always better medicine"}

Choosing Wisely Canada recommendations and lists are meant to spur conversations between patients and physicians to determine the appropriate course of care and treatment plan together. Each list contains tests, treatments and procedures within a specialty's scope of practice for which there is excellent evidence of overuse, waste or harm to patients. These lists are publicly available on the Choosing Wisely Canada website.

Specialty societies have used various processes for determining lists, but all have done so in accordance with the following principles:

1. The development process is thoroughly documented and publicly available.

2. Each recommendation is within the specialty's scope of practice.

3. Tests, treatments and procedures included are those that: (a) are frequently used, and (b) may expose patients to harm or stress.

4. Each recommendation is supported by evidence.

There is a growing international movement around this approach with Choosing Wisely programs being launched in 15 countries. An international working group, led by Choosing Wisely Canada, has articulated a set of five principles that should be incorporated into a Choosing Wisely campaign, which are to be physician-led, patientfocused, evidence-based, multi-professional and transparent (6).

\section{Antimicrobial stewardship and Choosing Wisely}

The management of infectious disease syndromes is often empiric, where treatment is initiated in the absence of full evidence, and on an educated clinical decision. In some circumstances, results may be pending at the time of initiation of antimicrobial therapy and in other cases; results may be negative when infection is in fact present. The wise choice of diagnostic testing-including when not to perform testing-is an important principle common to both Choosing Wisely Canada and the management of infectious diseases, to ensure rational and safe prescription of antimicrobial therapy (7).

Beyond judicious decisions around testing, there are additional principles of resource stewardship that have been a long-standing focus for infectious disease specialists (8). In an era of increasing antimicrobial resistance with limited availability of new and effective antibiotic agents, antimicrobial stewardship programs have emerged as a fundamental component of health care systems in Canada. Antimicrobial stewardship programs are one way that organizations and physicians are implementing the motivations behind Choosing Wisely into practice.

Antimicrobial stewardship programs have recently become a required organizational practice from Accreditation Canada. Evidence suggests that for Antimicrobial stewardship programs to be effective, local strategies need to be in place to ensure that this now mandated program has grassroots buy-in from frontline physicians (9). In contrast, Choosing Wisely Canada is fundamentally a grassroots campaign; nevertheless there is close alignment 
with the principles and goals of antimicrobial stewardship programs. Both programs aim to optimize patient outcomes while minimizing unintended harms associated with antimicrobial use, with the secondary goal of reducing health care costs without impacting quality.

Antimicrobial stewardship programs face similar challenges around changing medical practice as well as patient expectations. There is extensive literature regarding antimicrobial stewardship interventions and their impact on improving antimicrobial use, curbing antimicrobial resistance, and improving clinical outcomes $(10,11)$. Despite this, overuse of antimicrobials and their associated adverse effects remains a major day-to-day challenge.

Antimicrobial prescribing is a behaviour that is influenced by many complex social and cultural determinants, as well as ingrained individual behaviours that have typically been influenced by perceived patient expectations and other factors (12).

Choosing Wisely Canada offers an additional forum to facilitate conversations between physicians and patients around antimicrobial prescribing practices. Table 1 below demonstrates a selection of recommendations from Canadian and American specialty society Choosing Wisely lists around unnecessary or inappropriate antimicrobial use within the purview of their clinical practice. Many of these examples of antimicrobial overuse relate to outpatient practice, where antimicrobial stewardship has historically been more challenging to implement (13). Choosing Wisely recommendations focused on antibiotic use provide an opportunity to foster broader discussions around appropriate antimicrobial use, beyond what can be addressed by hospital antimicrobial stewardship programs.

Table 1: Examples of Canadian and American specialty society Choosing Wisely recommendations on antimicrobials

\begin{tabular}{|c|c|}
\hline Groups & Recommendations \\
\hline \multicolumn{2}{|r|}{ Canadian Specialty Society } \\
\hline Canadian Geriatrics Society & $\begin{array}{l}\text { Don't use antimicrobials to treat bacteriuria in older adults unless specific urinary } \\
\text { tract symptoms are present. }\end{array}$ \\
\hline $\begin{array}{l}\text { The College of Family Physicians of } \\
\text { Canada }\end{array}$ & $\begin{array}{l}\text { Don't use antibiotics for upper respiratory infections that are likely viral in origin, } \\
\text { such as influenza-like illness, or self-limiting, such as sinus infections of less than } \\
\text { seven days of duration. }\end{array}$ \\
\hline Canadian Urological Society & Don't use antimicrobials to treat asymptomatic bacteriuria in the elderly. \\
\hline Groups & Recommendations \\
\hline \multicolumn{2}{|r|}{ American Specialty Society } \\
\hline American Academy of Dermatology & $\begin{array}{l}\text { Don't prescribe oral antifungal therapy for suspected nail fungus without } \\
\text { confirmation of fungal infection. }\end{array}$ \\
\hline American Academy of Dermatology & $\begin{array}{l}\text { Don't use oral antibiotics for treatment of atopic dermatitis unless there is clinical } \\
\text { evidence of infection. }\end{array}$ \\
\hline $\begin{array}{l}\text { American Academy of Family } \\
\text { Physicians }\end{array}$ & $\begin{array}{l}\text { Don't routinely prescribe antibiotics for acute mild-to-moderate sinusitis unless } \\
\text { symptoms last for seven or more days, or symptoms worsen after initial clinical } \\
\text { improvement. }\end{array}$ \\
\hline American Academy of Ophthalmology & Don't order antibiotics for adenoviral conjunctivitis (pink eye). \\
\hline
\end{tabular}




\section{Shared challenges and opportunities}

Choosing Wisely Canada and antimicrobial stewardship programs share many of the same motivations and challenges. In the context of scarce health care resources, both initiatives can be framed by critics as cost-cutting or rationing exercises, rather than efforts to improve quality of care (3). Choosing Wisely Canada and international campaigns have remained independent from government, and it's clear to all stakeholders that recommendations should not be used by decision makers to delist services or determine payment. In fact, such delisting would be difficult to implement given that recommendations are not "never" events and rely on clinician decision making. Additionally, there are measurement challenges associated with both efforts as it can be difficult to quantify harm that has been avoided, or "appropriate" care given. Data are vital to garnering support from physicians, funders and stakeholders for these initiatives and there are efforts underway to better measure and demonstrate the impact of restraint on patient outcomes.

In terms of opportunities, there is a great deal of alignment between the principles of Choosing Wisely Canada and antimicrobial stewardship programs. Similarly, there are many shared levers of change for these efforts, which are physician-led initiatives to change practice, avoid harm and improve stewardship of valuable health care resources. These efforts can in turn influence public and patient expectations, and help to foster a conversation to make smart and effective choices to ensure high quality care.

\section{Conflict of interest}

None

\section{Funding}

None

\section{References}

(1) Brennan TA, Leape LL, Laird NM, Hebert L, et al. Incidence of adverse events and negligence in hospitalized patients-Results of the Harvard Medical Practice Study. N Engl J Med. 1991;324:370-6.

(2) Baker GR, Norton PG, Flintoft V, et al. The Canadian Adverse Events Study: The incidence of adverse events among hospital patients in Canada. CMAJ. 2004;170(11):1678-86.

(3) Deyo R. Cascade effects of medical technology. Ann Rev Pub Health. 2002;23:22-44.

(4) Institute of Medicine (US) Roundtable on Evidence-Based Medicine. Yong PL, Saunders RS, Olsen LA, editors. The Healthcare Imperative: Lowering Costs and Improving Outcomes-Workshop Series Summary. Washington: National Academies Press (US); 2010. p. 1-9.

(5) Emery DJ, et al. Overuse of magnetic resonance imaging. JAMA Intern Med. 2013;173(9):823-5.

(6) Levinson W, Kallewaard M, Bhatia RS, et al. 'Choosing Wisely': A growing international campaign. BMJ Qual Saf. 2014 Dec 31. doi:10.1136/bmjqs-2014-003821

(7) Mandell, LA, Wunderink RG, Anzueto A, et al. Infectious Diseases Society of America/American Thoracic Society Consensus Guidelines on the Management of Community-Acquired Pneumonia in Adults. Clin Infect Dis. 2007;44:S27-71.

(8) Dellit T, Owens R, McGowan J, et al. Infectious Diseases Society of America and the Society for Healthcare Epidemiology of America Guidelines for Developing an Institutional Program to Enhance Antimicrobial Stewardship. Clin Infect Dis. 2006;44:159-77

(9) Bal AM, Gould IM. Antibiotic stewardship: Overcoming implementation barriers. Curr Opin Infect Dis. 2011;24(4):357-62.

(10) Cosgrove SE, Seo SK, Bolon MK, et al. Evaluation of postprescription review and feedback as a method of promoting rational antimicrobial use: A multicenter intervention. Infect Control Hosp Epidemiol. 2012;33(4):374-80.

(11) Elligsen M, Walker SA, Pinto R, Simor A, et al. Audit and feedback to reduce broad-spectrum antibiotic use among intensive care unit patients: A controlled interrupted time series analysis. Infect Control Hosp Epidemiol. 2012;33(4):354-61. 
13 | CCDR SUPPLEMENT - June 18, 2015 - Volume 41S-4

(12) Charani E, Castro-Sanchez E, Sevdalis N, Kyratsis Y, et al. Understanding the determinants of antimicrobial prescribing within hospitals: The role of "prescribing etiquette." Clin Infect Dis. 2013;57(2):188-96.

(13) Drekonia DM, Filice GA, Greer N, et al. Antimicrobial stewardship in outpatient settings: A systematic review. Infect Control Hosp Epidemiol. 2015;36(2):142-52. 